\title{
EUS-guided retrieval of a migrated transgastric pancreatic stent
}
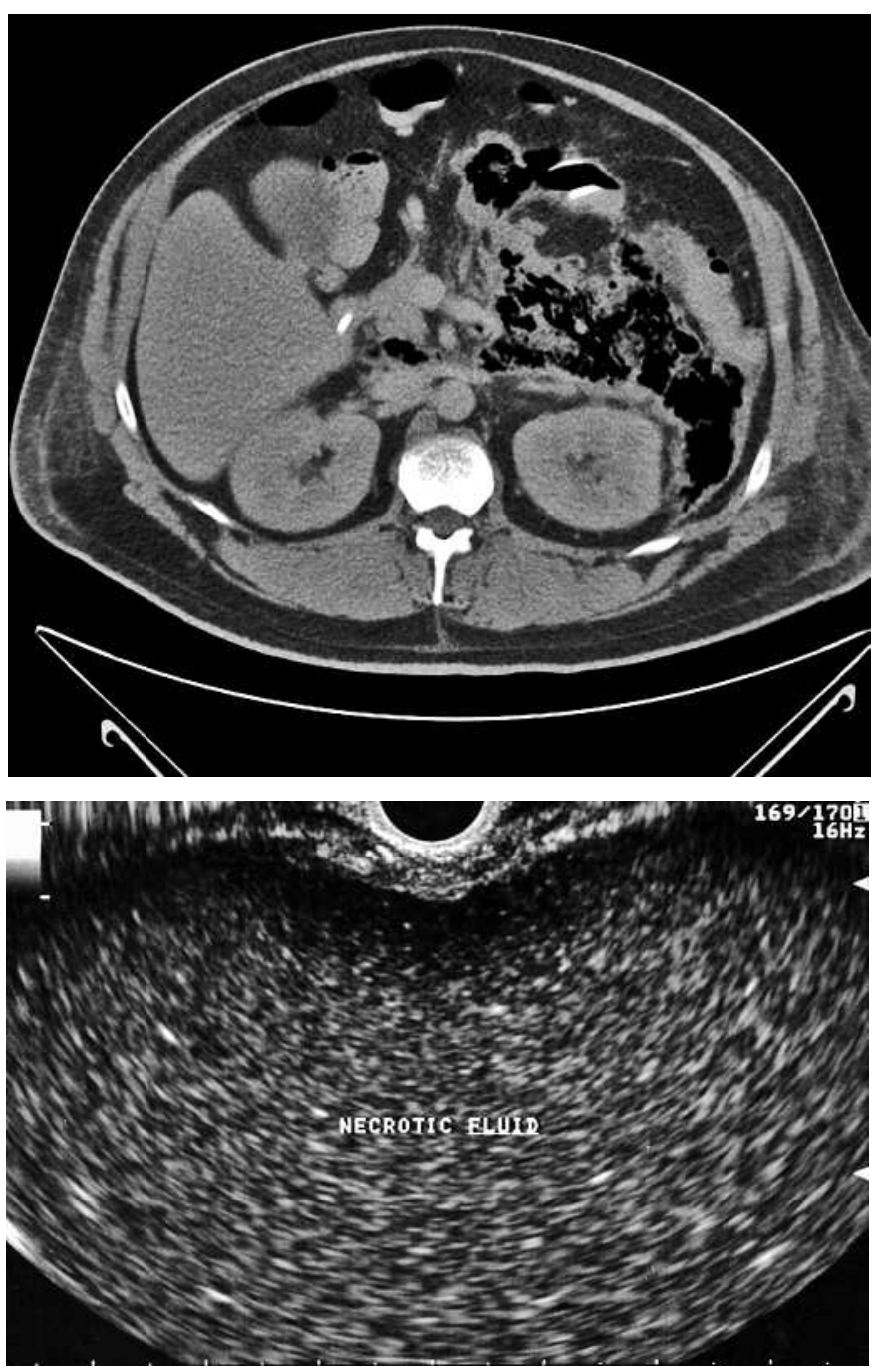

Figure 1 Abdominal computed tomography, revealing necrosis of the pancreas with extension to the iliac fossa and a migrated transgastric pancreatic stent.

Figure 2 Endoscopic ultrasonography using a curvilinear echo endoscope revealed a large pancreatic fluid collection with semisolid debris within it, consistent with pancreatic necrosis.
Transmural drainage of pancreatic pseudocysts has become an accepted alternative to surgery when an intervention is indicated. The complications of the procedure mainly involve bleeding, perforation, stent dysfunction, or migration into the gastrointestinal lumen [1]. We report here on a technique with which a stent that had migrated into the pancreatic necrosis cavity was retrieved successfully under endoscopic ultrasound (EUS) guidance.
A 48-year-old previously healthy patient was admitted with severe acute pancreatitis, and computed tomography (CT) revealed a pancreatic pseudocyst $15 \times 18 \mathrm{~cm}$ in size compressing the stomach. An endoscopic cystogastrostomy was carried out to relieve the gastric outlet obstruction. A repeat CT 1 week later revealed that the stent had migrated into the pseudocyst, possibly leading to necrosis (Figure 1). At gastroscopy, the gastric wall was diffusely edematous due to hy-

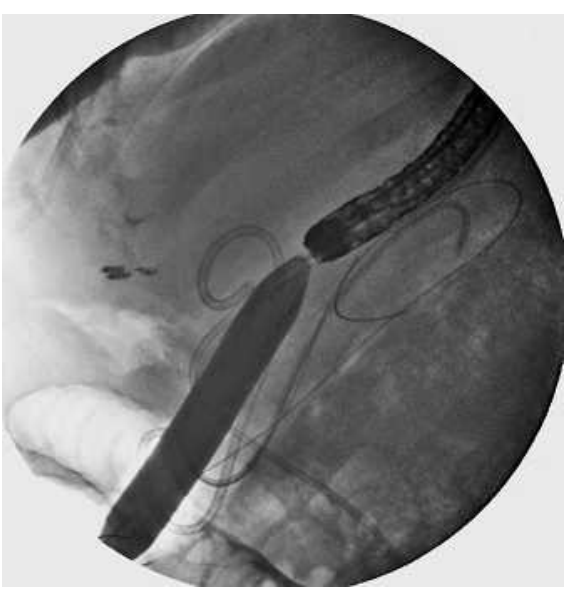

Figure 3 Dilation of the cystogastrostomy site to $60 \mathrm{Fr}$ was carried out under fluoroscopic guidance.

poalbuminemia (serum albumin $1 \mathrm{mg} /$ $\mathrm{dl}$ ), and a definitive area of luminal compression could not be identified. Subsequently, EUS revealed a large pancreatic fluid collection with semisolid debris, consistent with necrosis (Figure 2). Using a 19-gauge EUS-FNA needle (Echotip, Wilson-Cook GI Endoscopy, Winston-Salem, North Carolina, USA), the fluid collection was accessed and a 0.035-inch guide wire was coiled into the fluid collection under fluoroscopic guidance. The echo endoscope was then exchanged over a guide wire for a double-channel gastroscope. The stomach wall was then gradually dilated up to 60 Fr using over-thewire balloon dilators (Figure 3,4). After dilation, the necrotic cavity was intubated with the gastroscope, and the migrated stent was seen floating within the fluid collection. The stent was retrieved using rat-tooth forceps and positioned across the cystogastrostomy site (Figure $\mathbf{5 , 6}$ ). The patient was discharged after placement of a percutaneous jejunostomy feeding tube, with recommendations for outpatient follow-up. 


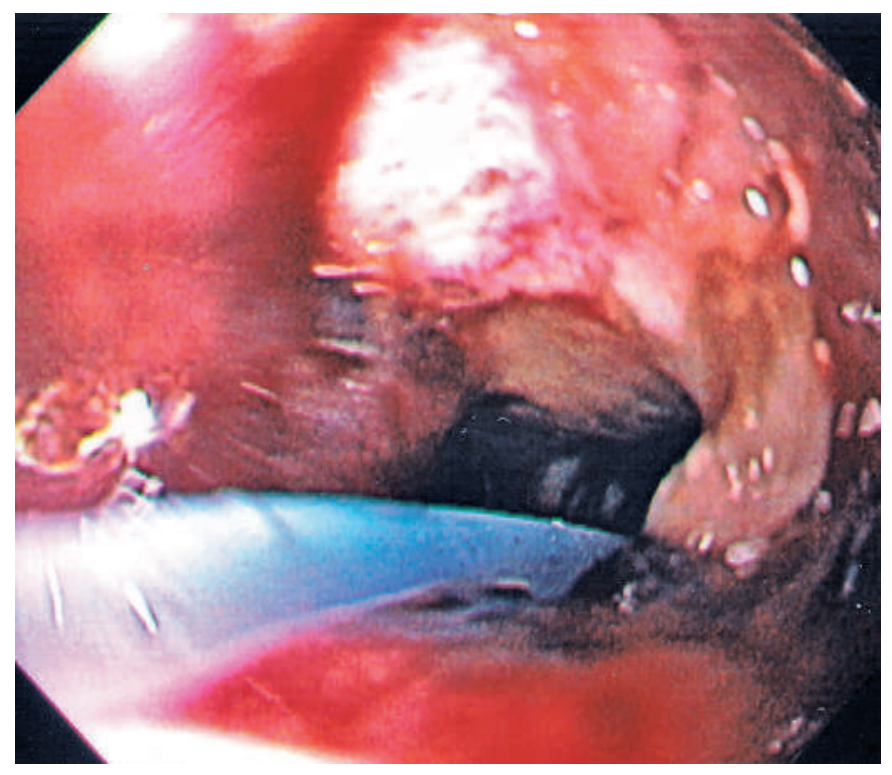

Figure 4 An adequate window is established between the stomach and the necrotic fluid collection to allow passage of a therapeutic gastroscope for stent retrieval.

Figure 5 The migrated stent, floating within the pancreatic fluid collection, is retrieved using a rat-tooth forceps.

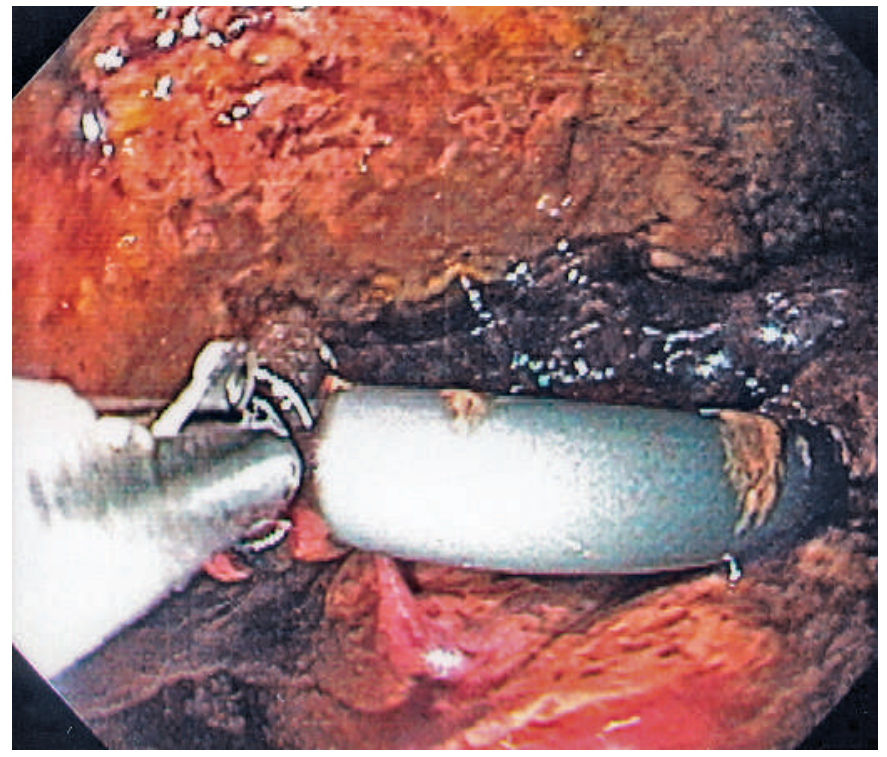

We are unaware of any prior reports of EUS being used for extraction of a migrated stent in a pancreatic fluid collection. The procedure is time-consuming, technically challenging, requires the use of multiple endoscopic accessories and fluoroscopy, and should only be undertaken with antibiotic coverage.

Endoscopy_UCTN_Code_TTT_1AS_2AD

\section{S. Varadarajulu}

Division of Gastroenterology and Hepatology, University of Alabama at Birmingham School of Medicine, Birmingham, Alabama, USA.

\section{Reference}

${ }^{1}$ Smits ME, Rauws EA, Tytgat GN, Huibregtse $K$. The efficacy of endoscopic treatment of pancreatic pseudocysts. Gastrointest Endosc 1995; 42: $202-207$

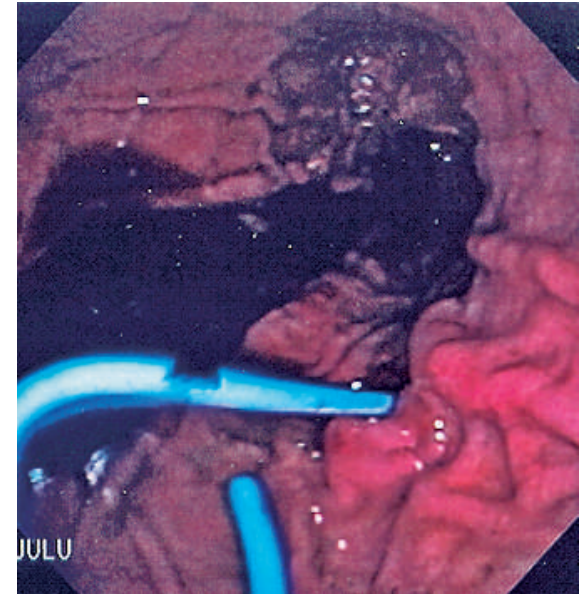

Figure 6 The stent is aligned properly across the cystogastrostomy site.

\section{Corresponding author}

\section{S. Varadarajulu, M.D.}

Division of Gastroenterology and Hepatology

University of Alabama at Birmingham Medical Center 410 LHRB 1530 Third Avenue South Birmingham, AL 35294

USA

Fax: +1-205-975-6381

Email: svaradarajulu@yahoo.com 